\title{
Aberrant expression of semaphorin 6B affects cell phenotypes in thyroid carcinoma by activating the Notch signalling pathway
}

\author{
Xiu-Juan Lv', Xin Chen', Yan Wang', Shan Yu', Lin Pang', Chao Huang ${ }^{2}$ \\ ${ }^{1}$ Department of Geriatric Medicine, The Fourth People's Hospital of Shenyang, Huanggu District, Shenyang, China \\ ${ }^{2}$ Department of Oncology, The Fourth People's Hospital of Shenyang, Huanggu District, Shenyang, China
}

\begin{abstract}
Introduction: Numerous semaphorins have been widely clarified to be involved in the development of multiple cancers. However, semaphorin 6B (SEMA6B) has not yet been extensively reported in cancers, especially in thyroid carcinoma.

Material and methods: Thyroid carcinoma RNA-Seq dataset from the TCGA database was used to assess the expression of SEMA6B in tissues, as well as its clinical significance. We adopted qRT-PCR and western blot analyses to measure the mRNA and protein expression of SEMA6B in thyroid carcinoma cells. The biological roles of SEMA6B in thyroid carcinoma cells were examined through cell counting kit 8, clone formation, and Transwell assays. Also, GSEA was used to identify the gene sets modulated by SEMA6B, which is further verified by western blot.

Results: According to the public dataset from the TCGA database, we found that the expression of SEMA6B was upregulated in thyroid carcinoma tissues compared to adjacent non-tumour tissues, and a high level of SEMA6B resulted in a poorer prognosis compared to the low-level SEMA6B group. Functional experiments showed that silencing SEMA6B suppressed the B-CPAP cells viability, invasiveness, and motility, whereas up-regulating SEMA6B in FTC-133 cells led to opposite outcomes. Furthermore, knockdown of SEMA6B in B-CPAP cells could significantly elevate the protein expression of NUMB and reduce the expression of NOTCH1, HES1, and Cyclin D1. Conversely, overexpression of SEMA6B in FTC-133 cells presented opposite results on the protein expression of these Notch signalling pathway-related markers.

Conclusions: Our findings demonstrated that SEMA6B exerts a tumourigenic effect in thyroid carcinoma partly by activating Notch signalling pathway, which provides a possible biomarker for the therapeutic intervention in thyroid carcinoma. (Endokrynol Pol 2021; 72 (1): 29-36)
\end{abstract}

Key words: semaphorin 6B; thyroid cancer; cell growth; invasion and migration; Notch signalling

\section{Introduction}

Thyroid carcinoma is a common malignant tumour in endocrine gland, which accounts for $94.5 \%$ of all endocrine tumours $[1,2]$. Statistics showed that the incidence of thyroid carcinoma is on the rise worldwide [3,4]. An American cancer report in 2019 estimated that there were 52,070 new cases of thyroid carcinoma, which ranks as the sixth most common malignancy in women [5]. In China, the national central cancer registry collected data from 449 registries across the country in 2014 and found that thyroid carcinoma ranked fourth among new malignancies in women [6]. The histopathological types of thyroid carcinoma includes follicular, poorly differentiated, papillary, anaplastic, and medullar thyroid carcinomas [7]. Among these types, papillary thyroid carcinoma is the most common, accounting for more than $80 \%$ of thyroid carcinomas [8]. Differentiated thyroid carcinomas (including papillary and follicular) have a low degree of malignancy and a good response to treatment [9]. However, some thyroid carcinomas with low differentiation or metastasis are still difficult to treat, showing the malignant characteristics of rapid tumour growth, high incidence of lymphatic metastasis, insensitivity to iodine treatment, and postoperative recurrence and metastasis [10]. Noticeably, a study reported that $67.9 \%$ of classic papillary thyroid carcinoma cases present with lymph node metastasis, which shows a higher recurrence rate and a higher disease-specific mortality compared with the intra-thyroid group [11, 12]. Therefore, early identification of papillary thyroid carcinoma and investigation of its potential mechanism are of great significance for the formulation of clinical comprehensive treatment plans and improvement of prognosis of papillary thyroid carcinoma patients.

Semaphores are a large family of secretory proteins and membrane-bound glycoproteins initially characterised as axonal guidance and neurodevelopment factors [13]. In recent years, in addition to being implicated in neural activity, semaphoring molecules have also been 
implicated in immune response and angiogenesis [14]. Also, increasing evidence has revealed that semaphores play a crucial role in the progression of tumours and are expected to be potential therapeutic bio-markers in various tumours [15]. The family of semaphores is divided into eight subtypes and contains more than 30 semaphore molecules of which subclasses 3-7 contain the 22 vertebrate semaphorins [16]. Class 6 Semaphorin $\mathrm{B}$ (SEMA6B) is a transmembrane protein belonging to the semaphorin family, which has been reported to participate in the progression of some cancers [17, 18]. It has been shown that SEMA6B levels were markedly elevated in gastric tumour cells in comparison with normal control cells, and silencing SEMA6B notably inhibited the cell invasion, migration, and adhesion in gastric cancer [17]. But in breast cancer tissues, 94\% of cancer tissues presented a lower expression of SEMA6B compared to the median value of normal tissues [18]. These two different kinds of findings illustrate that SEMA6B might play different roles in the progression of different tumours. But its role in thyroid carcinoma is still poorly understood.

The objective of this report was to examine the prognostic role of SEMA6B in thyroid carcinoma on the basis of the public database and to evaluate the effect of SEMA6B on the phenotype of thyroid carcinoma cells by in vitro experiments, as well as its influence on the Notch signalling pathway-related markers. All the data indicated that SEMA6B presents a prognostic value in thyroid carcinoma and may have the potential to be a useful bio-target for the treatment of thyroid carcinoma patients.

\section{Material and methods}

\section{Bioinformatics analysis}

The data for 568 cases including 510 thyroid carcinoma samples and 58 adjacent normal samples were retrieved from the RNA-Seq dataset of the TCGA (https://cancergenome.nih.gov/) database and were used for bioinformatics analysis.

Kaplan-Meier methods were used to evaluate the correlation between SEMA6B and the overall survival, which was compared by log-rank tests. The relevance between SEMA6B and clinical features was analysed using the chi-squared test.

\section{Cell culture}

The human papillary thyroid carcinoma cell lines B-CPAP and FTC133 were acquired from the Shanghai Chinese Academy of Sciences cell bank. The normal thyroid epithelial cells - Nthy-ori 3-1 - were acquired from European Collection of Cell Culture (Salisbury, UK). These three types of cells were incubated in RPMI-1640 medium (Beyotime, Jiangsu, China) supplemented with $10 \%$ foetal bovine serum (FBS) and antibiotics including penicillin $(100 \mathrm{U} / \mathrm{ml})$ and streptomycin $(0.1 \mathrm{mg} / \mathrm{ml})$ under a humidified atmosphere of $5 \%$ $\mathrm{CO}_{2}$ at $37^{\circ} \mathrm{C}$.

\section{Transfection}

Lipofectamine2000 (Invitrogen) was used following the manufacture's protocol. Two small interference RNAs (si-RNAs) targeting SEMA6B (si-SEMA6B-1: 5'-CCTAGCCATTGATGCTGTCAT-3', si-SEMA6B-2: 5'-CCGGATGACATCCTCAACTTT-3') were used to perform the loss-of-function of SEMA6B assay, and a scramble siRNA (si-con: 5' - CGAACUCACUGGUCUGACC $-3^{\prime}$ ) was used as negative control (NC). Plasmid pcDNA3.1-SEMA6B was used to implement the gain-of-function of SEMA6B assay, and pcDNA3.1 empty vector served as NC. All the siRNAs and plasmids were synthesised from the Shanghai GeneChem Corporation (China).

\section{Quantitative Reverse-Transcription PCR}

Total RNA was extracted from cultured cells using an Ultrapure RNA kit (ComWin Biotech, CWBIO, Beijing, China) and reverse transcribed to cDNA using the HiFiScript cDNA Synthesis Kit (CWBIO). The mRNA level of SEMA6B was measured by UltraSYBR Mixture (CWBIO). Primers are listed below:

- SEMA6B F: 5'-GTCGGAGACAACATCAGCGGTA-3',

- SEMA6B R: 5'-GCATCAATGGCTAGGAAGTCGG-3';

- GAPDH F: 5'-TGTGTCCGTCGTGGATCTGA-3',

- GAPDH R: 5'-CCTGCTTCACCACCTTCTTGA-3'.

Relative mRNA level of SEMA6B was evaluated using the comparative $\mathrm{Ct}$ value and normalised to GAPDH.

\section{Western blot}

Total proteins were extracted using RIPA buffer (CWBIO) with proteinase inhibitor from cells after transfection for $24 \mathrm{~h}$ and quantified using the bicinchoninic acid disodium assay. Then, 20 $\mu \mathrm{g}$ of proteins were loaded onto $10 \%$ SDS-PAGE and transferred onto PVDF membrane. Upon blocking with 5\% non-fat dried milk for an hour, the membrane was subjected with primary antibodies anti-SEMA6B (PA5-47251, Invitrogen, Carlsbad, CA, USA), anti-NUMB (PA5-81233, Invitrogen), anti-NOTCH1 (PA5-32522, Invitrogen), anti-HES1 (PA5-28802, Invitrogen), anti-Cyclin D1 (MA5-16356, Invitrogen), and GAPDH rabbit polyclonal antibody (PA1-16777, Invitrogen) at $4^{\circ} \mathrm{C}$ for 24 hours. After rinsing three times with TBST, the membranes were subjected with horseradish peroxidase-labelled secondary antibodies for an hour. The labelled bands were determined using SuperSignal chromogenic reagent kit (Beyotime, Nantong, China) and quantified by Image J 1.44 software (NIH, Bethesda, MD, USA).

\section{Cell proliferation assay}

For cell counting kit 8 (CCK8) assay, transfected B-CPAP and FTC133 cells at a density of $0.1 \times 10^{4}$ cells/well were seeded in each well of 96-well plates and incubation for 72 hours. At the points of $0 \mathrm{~h}$, $24 \mathrm{~h}, 48 \mathrm{~h}$, and $72 \mathrm{~h}, 10 \mu \mathrm{L}$ CCK8 reagent (Beyotime) was added to each well and cultured for another $1.5 \mathrm{~h}$. The optical density (OD) value was determined using an Elx800 Reader (Bio-Tek, Winooski, VT, USA) according to the manufacturer's instructions.

For clone formation assay, transfected cells (500 cells/dish) were seeded in a $60 \mathrm{~mm}$ dish and cultured at $37^{\circ} \mathrm{C}$ with $5 \% \mathrm{CO}_{2}$ for 1-2 weeks until cells formed sufficiently large colonies. Then, the colonies were fixed with $5 \mathrm{ml} 4 \%$ paraformaldehyde for 30 minutes and stained with $0.1 \%$ crystal violet for 20 minutes. After air-drying, colonies were manually counted and photographed.

\section{Cell invasion and migration assay}

Transwell invasion and migration assay was conducted using 24-well Transwell plates (Corning Inc., Corning, NY, USA) with or without Matrigel-coated membrane, respectively. Transfected cells $\left(1 \times 10^{4}\right.$ for invasion assay and $0.5 \times 10^{4}$ for migration assay) suspended in $100 \mu \mathrm{l}$ serum-free RPMI-1640 medium were seeded into the upper compartment, while $500 \mu \mathrm{l}$ RPMI-1640 plus 10\% FBS was filled in the lower compartment. Upon cultivation at $37^{\circ} \mathrm{C}$ for $24 \mathrm{~h}$, cells that had invaded or migrated through the $8-\mu \mathrm{m}$ pores 

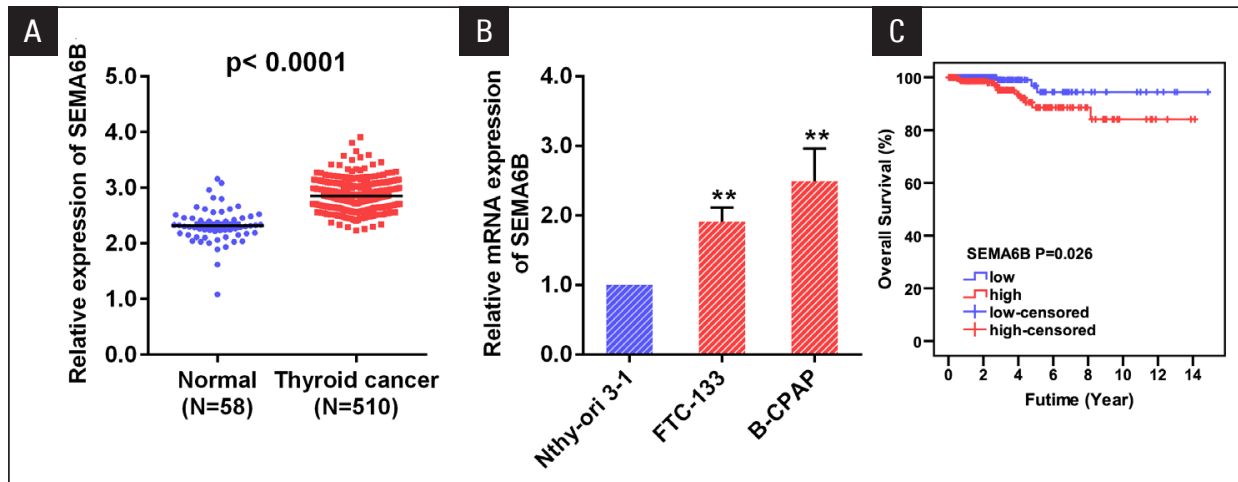

Figure 1. $m R N A$ levels of SEMA6B in thyroid carcinoma and survival curve. A. SEMA6B expression in thyroid carcinoma tissues and the adjacent non-tumour tissues in the thyroid carcinoma RNA sequencing dataset from the TCGA database. B. QRT-PCR analysis of $m R N A$ level of SEMA6B in malignant thyroid carcinoma B-CPAP and FTC-133 cells by comparison with non-malignant thyroid epithelial Nthy-ori 3-1 cells. ${ }^{*} p<0.01$, compared with Nthy-ori 3-1. C. The overall survival curve was plotted by Kaplan-Meier methods based upon the expression of SEMA6B in thyroid carcinoma patients in the TCGA cohort

were rinsed with PBS, fixed using paraformaldehyde, and stained by crystal violet. Next, the number of invaded and migrated cells was counted in five representative fields under light microscopy $(40 \times$ magnification) and photographed.

\section{Statistical analyses}

All the statistical analyses were adopted using GraphPad Prism 6.0 software (San Diegl, CA, USA) and SPSS 22.0 software (IBM SPSS, Armonk, NY, USA). Differences between two groups was determined by unpaired Student's $t$ test, and the differences between multiple groups were compared by one-way analysis of variance (ANOVA) followed a post hoc test. All the experiments were conducted in triplicate. A p value of less than 0.05 was defined as statistical significance.

\section{Results}

\section{SEMA6B expression is elevated in thyroid carcinoma and leads to a poor prognosis}

To get an overview of SEMA6B status in thyroid carcinoma, we first analysed the expression of SEMA6B in the RNA-Seq dataset of thyroid carcinoma from the TCGA database and observed that SEMA6B was significantly upregulated in thyroid carcinoma tissues in comparison with the adjacent non-tumour tissues ( $p<0.0001$, Fig 1A). Next, we selected two commonly used thyroid carcinoma cell lines to detect the mRNA level of SEMA6B by qRT-PCR. Consistent with the SEMA6B expression in tissues, the mRNA levels of SEMA6B were obviously elevated in thyroid carcinoma cells in contrast to normal thyroid epithelial cells ( $p<0.01$, Fig. 1B), suggesting that the upregulated SEMA6B might be involved in the development of thyroid carcinoma.

Next, to determine the clinical significance of SEMA6B in thyroid carcinoma, we evaluated the correlation between SEMA6B and clinical characteristics based upon the public dataset from the TCGA database. The data were divided into high and low groups accord-
Table 1. Correlation between clinical features of thyroid carcinoma and the expression of SEMA6B in the TCGA cohort

\begin{tabular}{lccc}
\hline \multirow{2}{*}{ Characteristics } & \multicolumn{2}{c}{ Expression of SEMA6B } & \multirow{2}{*}{ p value } \\
\cline { 2 - 3 } & Low & High & \\
\hline Age (years) & & & 0.192 \\
\hline$<45$ & 108 & 94 & \\
\hline$\geq 45$ & 127 & 141 & \\
\hline Gender & & & 0.677 \\
\hline Female & 174 & 170 & \\
\hline Male & 61 & 65 & \\
\hline Stage & & & 0.771 \\
\hline I + II & 155 & 152 & \\
\hline III + IV & 80 & 83 & \\
\hline Pathologic-T & & & 0.850 \\
\hline T1 + T2 & 143 & 141 & \\
\hline T3 + T4 & 92 & 94 & \\
\hline Pathologic-N & & & 0.406 \\
\hline N0 & 120 & 129 & \\
\hline N1 & 115 & 106 & \\
\hline Pathologic-M & & & $0.044^{*}$ \\
\hline M0 & 227 & 217 & \\
\hline M1 & 8 & 18 & \\
\hline P $~$ & & & \\
\hline
\end{tabular}

${ }^{*} p<0.05$

ing to the median expression of SEMA6B and showed that SEMA6B expression had a significant correlation with pathologic metastasis $(\mathrm{M})(\mathrm{p}<0.05$, Tab. 1), indicating that SEMA6B might play a role in promoting metastasis in thyroid carcinoma. Kaplan-Meier method revealed that low SEMA6B expression has a higher probability of a better overall survival rate compared to the high SEMA6B expression group ( $p<0.01$, Fig. 1C). 
All these data illustrated that SEMA6B may be a useful bio-target for the diagnosis and prognosis of thyroid carcinoma.

\section{SEMA6B enhances cell viability in thyroid carcinoma}

To better understand the biological functions of SEMA6B in thyroid carcinoma cells, we implemented CCK8 and clone formation assays to detect the cell viability after altering the expression of SEMA6B. Before detecting, we established B-CPAP cells with depleted SEMA6B using siRNA targeting SEMA6B and FTC-133 cells with overexpressed SEMA6B using pcDNA3.1-SEMA6B. The expression of SEMA6B was measured using qRT-PCR and western blot analyses. The data revealed that the mRNA and protein expression of SEMA6B was notably reduced upon knockdown of SEMA6B, whereas SEMA6B expression was dramatically elevated after

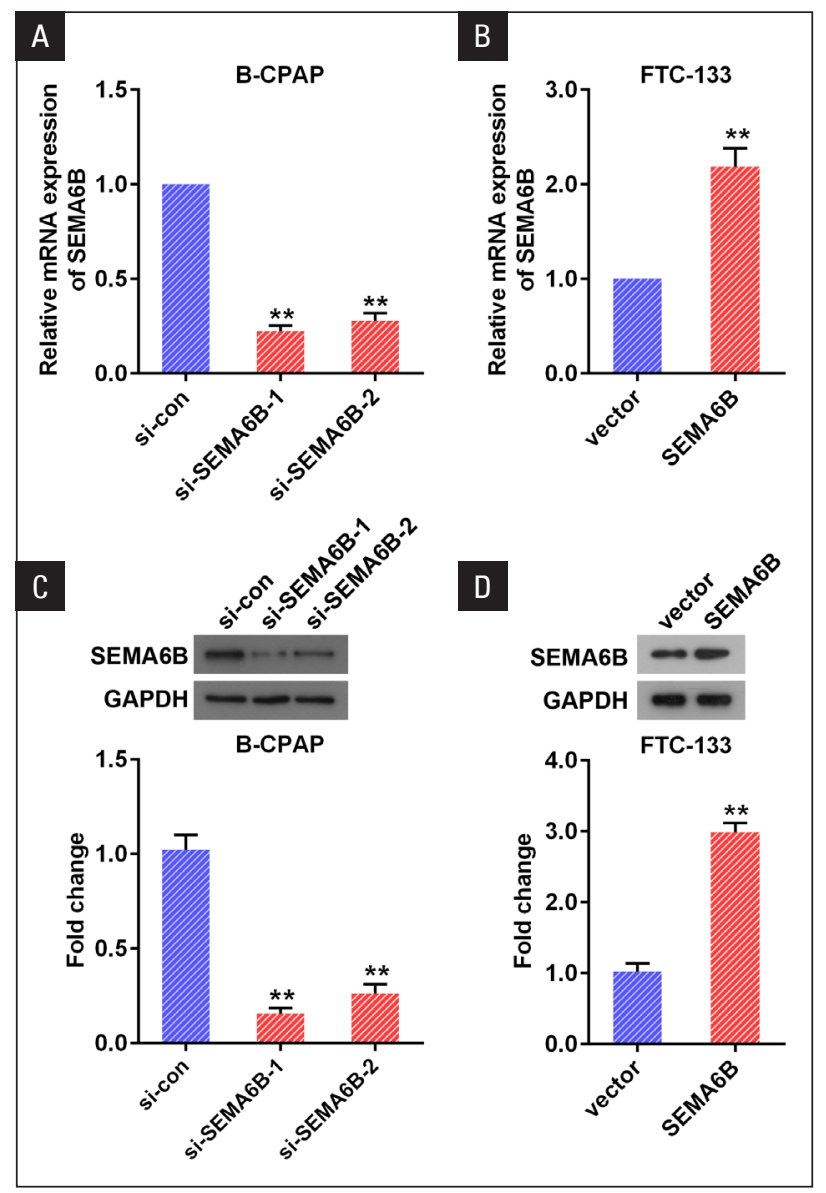

Figure 2. Transfection efficiency of loss- and gain-of-function of $S E M A 6 B$. A. C. Detection of transfection efficiency in B-CPAP cells which were treated with si-con, si-SEMA6B-1, and siSEMA6B-2. B. D. Detection of transfection efficiency in FTC-133 cells that were with $p c D N A 3.1$ empty vector, pcDNA3.1-SEMA6B. The expression of SEMA6B was determined using $q R T-P C R(\mathbf{A}, \mathbf{B})$ and western blot $(\mathbf{C}, \mathbf{D}) .{ }^{* *} p<0.01$, compared with the si-con group or vector overexpression of SEMA6B compared to their respective controls ( $p<0.01$, Fig. 2). For the loss-of-function of SEMA6B, si-SEMA6B-1 was more efficient, so it was selected to perform the further knockdown tests. The CCK8 assays demonstrated that knockdown of SEMA6B led to an impaired proliferation in B-CPAP cells in comparison with the si-con group ( $p<0.05$, Fig. $3 \mathrm{~A}$ ); however, the enhanced expression of SEMA6B resulted in an elevated proliferation in FTC-133 cells in contrast to the pcDNA3.1 empty vector group ( $p<0.01$, Fig. 3B). Additionally, the significant difference of the knockdown and overexpression tests first appeared at the $24 \mathrm{~h}$ and $48 \mathrm{~h}$ points, respectively. The effects of SEMA6B on the cell viability were also confirmed by clone formation assay, which indicated that the colony-forming capacity was dramatically reduced in B-CPAP cells with depleted SEMA6B ( $p<0.01$, Fig. 3C-D), while overexpression of SEMA6B drastically increased the number of colonies in FTC-133 cells ( $p<0.01$, Fig. 3E-F). Collectively, all these results illustrated that SEMA6B exerts a pro-proliferative effect in thyroid carcinoma cells.

\section{SEMA6B promotes cell invasion and migration in thyroid carcinoma}

To examine the potential effect of SEMA6B on the invasiveness and motility, Transwell assay was implemented. As shown in Figure 4A, silencing SEMA6B inhibited the invasive and migratory capacities of B-CPAP cells by comparison to the si-con group ( $p<0.01$, Fig. 4A-B). On the other hand, upregulating SEMA6B enhanced the invasive and migratory abilities of FTC-133 cells in contrast to the vector group ( $p<0.01$, Fig. 4C-D). Taken together, these observations demonstrated that SEMA6B could affect the cell invasion and migration in thyroid carcinoma.

\section{SEMA6B activates the Notch pathway}

Studies have shown that the Notch pathway is involved in the regulation of the recurrence and development of a variety of tumours, and it plays a role in synergistic or antagonistic way [19]. In our work, GSEA revealed that high level of SEMA6B positively correlated with the Notch signalling pathway ( $\mathrm{p}<0.01$, Fig. 5A). Thus, to verify the correlation between SEMA6B and Notch pathway, we detected the protein expression of Notch signalling-related markers, including antagonist NUMB, NOTCH1, downstream target HES1, and growth-promoting gene Cyclin D1, by gain- and loss-of-function of SEMA6B assays. The data showed that, compared to the si-con group, silencing SEMA6B significantly elevated the expression of NUMB and reduced the expression of NOTCH1, HES1, and Cyclin D1 ( $p<0.01$, Fig. 5B and C). Conversely, overexpression of SEMA6B resulted in a dramatic decrease in the 


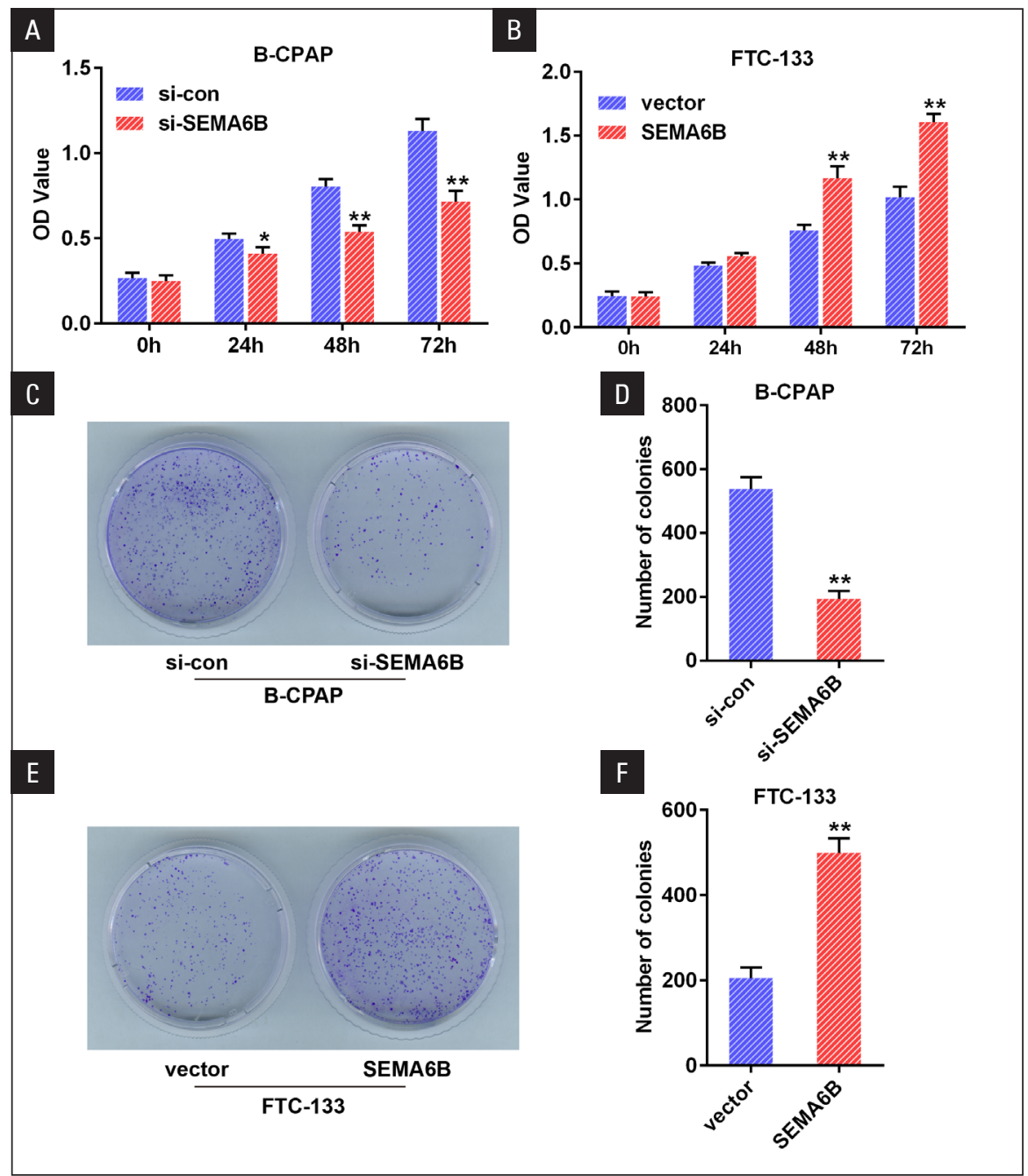

Figure 3. Effects of SEMA6B on the cell viability in thyroid carcinoma. A, B. CCK8 assays were used to determine the OD values when silencing SEMA6B in B-CPAP cells (A) and upregulation of SEMA6B in FTC-133 cells (B). C-F. Clone formation assays were implemented to examine the cells colony-forming abilities when silencing SEMA6B in B-CPAP cells $(\mathbf{C}, \mathbf{D})$ and upregulation of SEMA6B in FTC-133 cells (E, F). (C, E) Representative colonies in dishes. (D, F) The number of colonies was quantified. ${ }^{* *} p<0.01$, compared with si-con group or vector

expression of NUMB but led to an obvious increase on the protein level of NOTCH1, HES1, and Cyclin D1 $(\mathrm{p}<0.01$, Fig. 5B, D). Thus, we inferred that SEMA6B plays a promoting effect in thyroid carcinoma partly by activating the Notch signalling pathway.

\section{Discussion}

The proteins galectin-3, cytokeratin-19, and HBME-1 are the most studied and meaningful markers associated with thyroid carcinoma diagnosis and prognosis [20]. However, at present there is no specific and sensitive marker for papillary thyroid carcinoma. Therefore, more meaningful biomarkers associated with the development of papillary thyroid carcinoma need to be identified. Our work focused on the evaluation of the role of SEMA6B in the diagnosis and prognosis of thyroid carcinoma and the exploration of its functional roles, as well as the underlying mechanism, hoping to provide a possible marker for the therapy of thyroid carcinoma.

For semaphorins, the class 3 and 4 family members have been well described in the progression of cancers. Numerous studies have clarified that some semaphorins, such as SEMA3F [21, 22] and SEMA3B [23], function as inhibitors to suppress tumour progression, while other semaphorins, such as SEMA4A [24], SEMA4D [25], SEMA5A [26], and SEMA6A [27], function as promoters to facilitate tumour progression. SEMA6B, a class 6 semaphorin, was first observed at a higher level in normal brain tissues and at a lower level in other tissues [28]. However, its role in cancers is rarely reported. In the current study, by bioinformatics analysis and qRT-PCR detection, SEMA6B was shown 


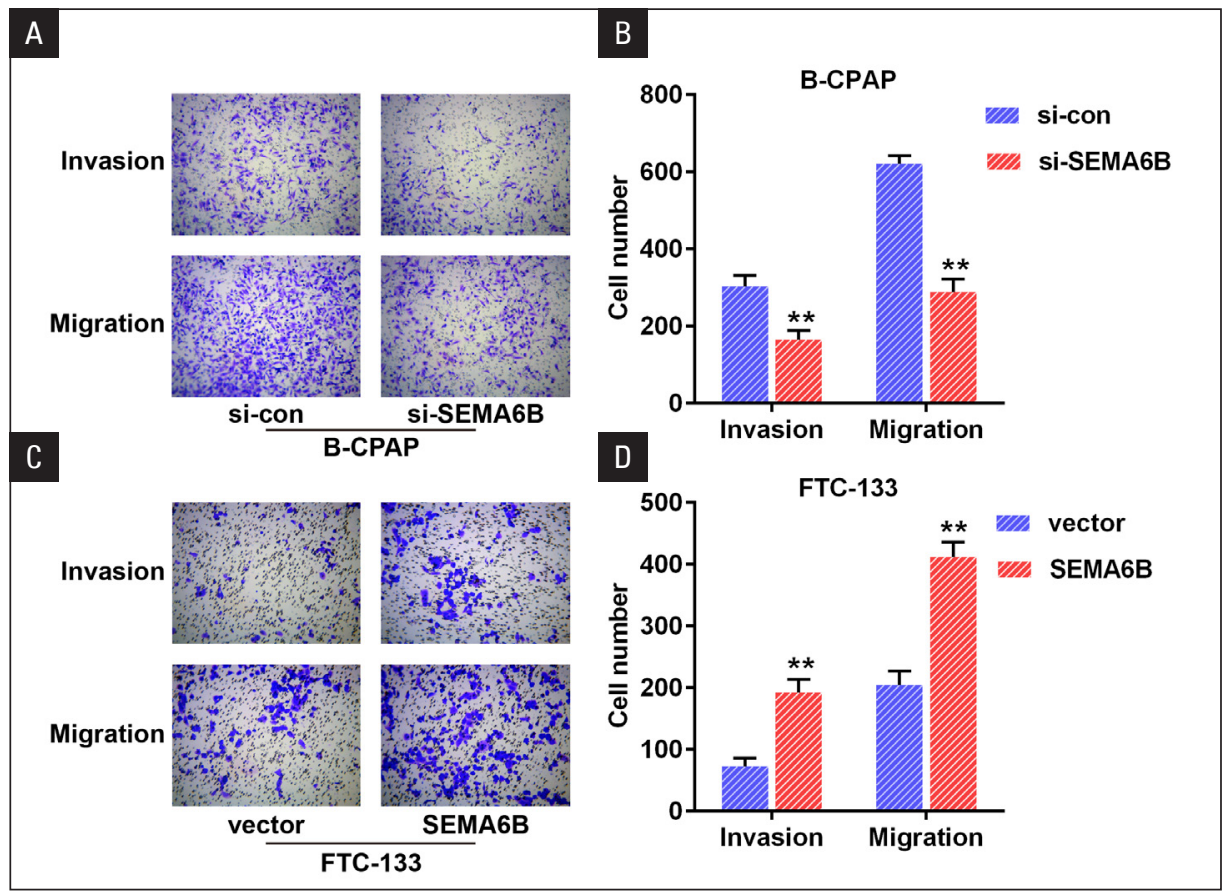

Figure 4. Effects of SEMA6B on the cell invasiveness and motility in thyroid carcinoma. Transwell assays were implemented to determine the invasion and migration of B-CPAP cells with knockdown of SEMA6B (A, B) and FTC-133 cells with overexpression of SEMA6B (C, D). (A, C) Representative photographs of invasion and migration cells. (B, D) The number of invaded and migrated cells was quantified. ${ }^{* *} p<0.01$, compared with the si-con group or vector

to be highly expressed in thyroid carcinoma and led to a poorer survival, hinting that SEMA6B may play a role in tumourigenesis in thyroid carcinoma. Consistent with these findings, the mRNA and protein levels of SEMA6B were also found to be higher in gastric cancer cell line SGC-7901 in contrast to normal cells. Importantly, the functional experiments showed that inhibition of SEMA6B expression notably suppressed the gastric cancer cell invasion, migration, and adhesion [17]. Coincidentally, using thyroid cells, we also found that SEMA6B could dramatically enhance the cell viability, invasiveness, and motility. Interestingly, the reduced expression of SEMA6B in breast cancer in contrast to healthy samples was frequently detected [18]. SEMA6B is not the only one showing this duality. Another member of class 6, SEMA6A, was reported to inhibit glioma cells proliferation, invasion, and migration [29], while its expression was elevated in several renal tumour tissues compared to adjacent normal tissues [30]. The mechanisms responsible for this duality are not fully understood. One article explains that this may be the result of post-translational processing and the formation of complex associations between semaphorin receptors and other types of membrane-bound receptors [14]. However, further research is needed.

Abnormal Notch signalling pathway appears in many malignant tumours [31]. Thyroid carcinoma is no exception. Aberrant Notch pathway has been found to inhibit or promote tumour progression depending on the cell type and environment [32]. NOTCH1 is one of the four receptors of Notch signalling pathway, and its role in papillary thyroid carcinoma and related mechanisms are still controversial. At present, most scholars consider that NOTCH1 signalling pathway can promote papillary thyroid carcinoma cell proliferation and metastasis, and is closely linked to worse prognosis. Piana et al. found that positivity of NOTCH1 was predominantly correlated with papillary thyroid carcinoma by immunohistochemistry through a series of 106 thyroid specimens, suggesting that the progression of papillary thyroid carcinoma is positively correlated with the activation of NOTCH1 [33]. By GSEA analysis, we found that high expression of SEMA6B has a positive correlation with markers related to Notch signalling pathway, which raises the question of whether the growth- and invasive-promoting effects of SEMA6B in thyroid carcinoma are correlated with NOTCH1 protein. Moreover, NUMB as a Notch signalling antagonist inhibits Notch signalling by stabilising the intracellular domain of NOTCH1 receptor, suggesting that the expression of NUMB should be inversely proportional to the expression of SEMA6B [34]. In addition, as a downstream target gene of Notch pathway, HES1 transmits down Notch signal and can maintain a variety of immature cells in an undifferentiated state [35]. Also, studies have reported that the activation of 
A

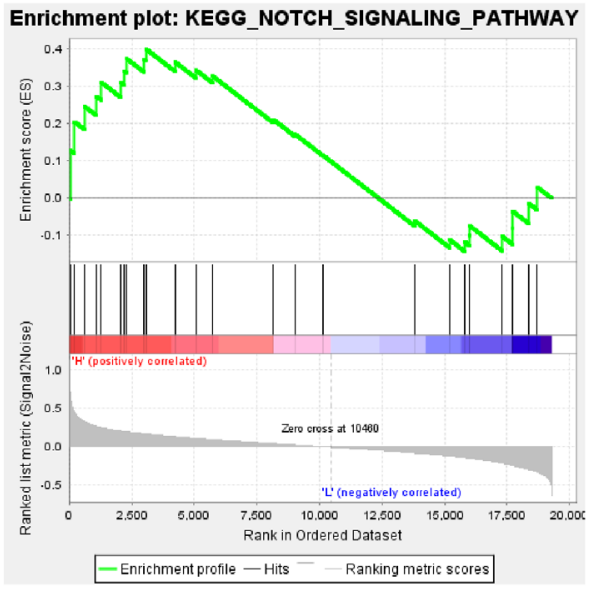

B

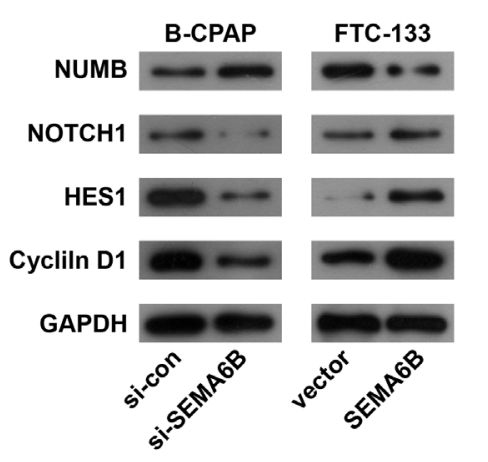

C

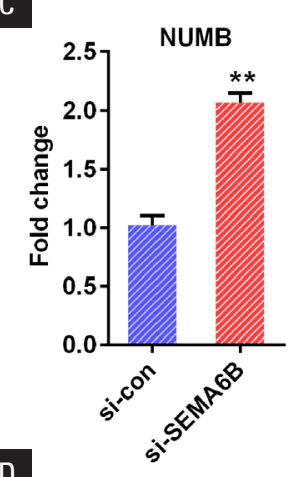

D

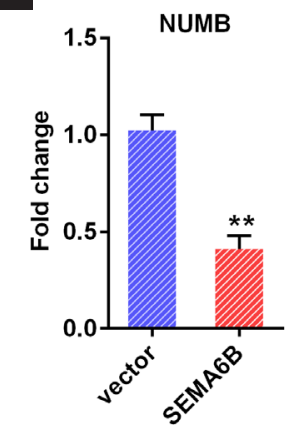

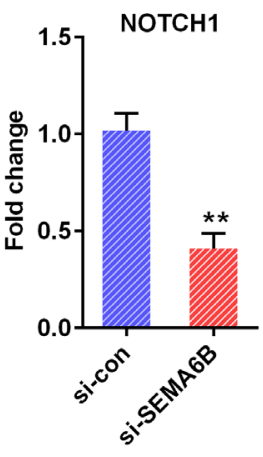

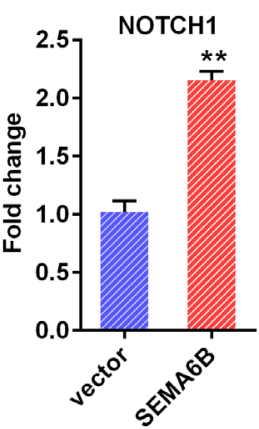

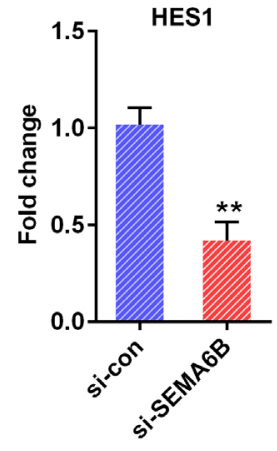
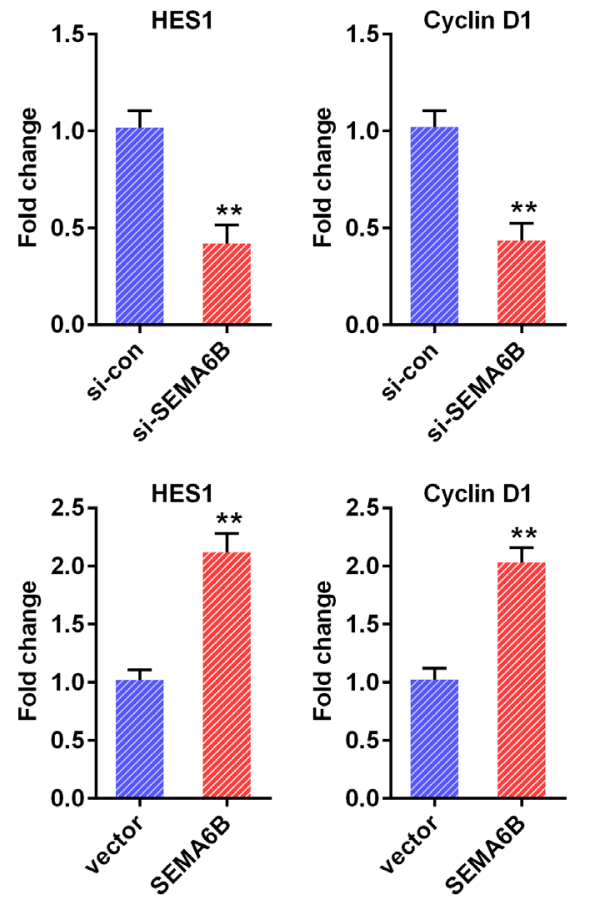

Figure 5. Correlation between SEMA6B and the Notch signalling relative proteins. A. GSEA analysis showed the enrichment of the Notch pathway-related markers in the high SEMA6B expression group based on the TCGA database. B. Protein levels of NUMB, NOTCH1, HES1, and Cyclin D1 in B-CPAP and FTC-133 cells after altering the expression of SEMA6B by western blot analysis. C, D. The greyscale values were quantified. ${ }^{* *} p<0.01$, compared with the si-con group or vector

Notch pathway induces growth-promoting genes such as cyclin D1 to cause tumour formation, so we explored whether SEMA6B induces cyclin D1 to participate in the proliferation of thyroid carcinoma cells [36]. Western blot analysis revealed that silencing SEMA6B notably reduced the protein levels of NOTCH1, HES1, and cyclin D1, accompanied by an increase in HES1 protein expression, while overexpression of SEMA6B gave the opposite results. Therefore, it is reasonable to speculate that SEMA6B may play a tumourigenic effect in thyroid carcinoma by activating the Notch signalling pathway.

\section{Conclusions}

Taken together, the current study found that SEMA6B has a prognostic value in thyroid carcinoma and suggested that during the progression and tumourigenesis of thyroid carcinoma, SEMA6B promoted the cell proliferation, migration, and invasion partly through regulating the Notch signalling pathway. These results highlight the potential of SEMA6B to be a target for the therapeutic intervention of thyroid carcinoma. 


\section{References}

1. Zhang H, Hu N. Telomerase reverse transcriptase induced thyroid carcinoma cell proliferation through PTEN/AKT signaling pathway. Mol Med Rep. 2018; 18(2): 1345-1352, doi: 10.3892/mmr.2018.9119, indexed in Pubmed: 29901196.

2. Piraino P, Sepúlveda A, Lillo R, et al. [Thyroid cancer. Report of 85 cases]. Rev Med Chil. 2000; 128(4): 405-410, indexed in Pubmed: 10962858.

3. Wang Y, Wang Y, Wang W. Increasing incidence of thyroid cancer in shanghai, China, 1983-2007. Nature Precedings. 2011, doi: 10.1038/npre.2011.5994, indexed in Pubmed: 22345304.

4. Chen AY, Jemal A, Ward EM. Increasing incidence of differentiated thyroid cancer in the United States, 1988-2005. Cancer. 2009; 115(16): 3801-3807, doi: 10.1002/cncr.24416, indexed in Pubmed: 19598221.

5. DeSantis CE, Ma J, Gaudet MM, et al. Cancer statistics, 2019. CA Cancer J Clin. 2019; 69(1): 7-34, doi: 10.3322/caac.21551, indexed in Pubmed: 30620402.

6. Chen WQ, Li H, Sun KX, et al. [Report of Cancer Incidence and Mortality in China, 2014]. Zhonghua Zhong Liu Za Zhi. 2018; 40(1): 5-13, doi: 10.3760/cma.j.issn.0253-3766.2018.01.002, indexed in Pubmed: 29365411.

7. Chmielik E, Rusinek D, Oczko-Wojciechowska M, et al. Heterogeneity of Thyroid Cancer. Pathobiology. 2018; 85(1-2): 117-129, doi: 10.1159/000486422, indexed in Pubmed: 29408820.

8. Cabanillas M, McFadden D, Durante C. Thyroid cancer. Lancet. 2016; 388(10061): 2783-2795, doi: 10.1016/s0140-6736(16)30172-6, indexed in Pubmed: 27240885.

9. Kumar A, Bal CS. Differentiated thyroid cancer. Indian J Pediatr. 2003; 70(9): 707-713, doi: 10.1007/BF02724312, indexed in Pubmed: 14620185.

10. Sampson E, Brierley JD, Le LW, et al. Clinical management and outcome of papillary and follicular (differentiated) thyroid cancer presenting with distant metastasis at diagnosis. Cancer. 2007; 110(7): 1451-1456, doi: 10.1002/cncr.22956, indexed in Pubmed: 17705176.

11. Smith VA, Sessions RB, Lentsch EJ. Cervical lymph node metastasis and papillary thyroid carcinoma: does the compartment involved affect survival? Experience from the SEER database. J Surg Oncol. 2012; 106(4): 357-362, doi: 10.1002/jso.23090, indexed in Pubmed: 22392921.

12. Liu FH, Kuo SF, Hsueh C, et al. Postoperative recurrence of papillary thyroid carcinoma with lymph node metastasis. J Surg Oncol. 2015; 112(2): 149-154, doi: 10.1002/jso.23967, indexed in Pubmed: 26175314.

13. Alto LT, Terman JR. Semaphorins and their Signaling Mechanisms. Methods Mol Biol. 2017; 1493: 1-25, doi: 10.1007/978-1-4939-6448-2 1, indexed in Pubmed: 27787839.

14. Neufeld G, Mumblat $Y$, Smolkin T, et al. The role of the semaphorins in cancer. Cell Adh Migr. 2016; 10(6): 652-674, doi: 10.1080/19336918.2016. 1197478, indexed in Pubmed: 27533782.

15. Gurrapu S, Tamagnone L. Transmembrane semaphorins: Multimodal signaling cues in development and cancer. Cell Adh Migr. 2016; 10(6): 675-691, doi: 10.1080/19336918.2016.1197479, indexed in Pubmed: 27295627.

16. Gu C, Giraudo E. The role of semaphorins and their receptors in vascular development and cancer. Exp Cell Res. 2013; 319(9): 1306-1316, doi: 10.1016/j.yexcr.2013.02.003, indexed in Pubmed: 23422037.

17. Ge C, Li Q, Wang $\mathrm{L}$, et al. The role of axon guidance factor semaphorin $6 \mathrm{~B}$ in the invasion and metastasis of gastric cancer. J Int Med Res. 2013; 41(2): 284-292, doi: 10.1177/0300060513476436, indexed in Pubmed: 23781008.

18. D'Apice L, Costa V, Valente C, et al. Analysis of SEMA6B gene expression in breast cancer: identification of a new isoform. Biochim Biophys Acta. 2013; 1830(10): 4543-4553, doi: 10.1016/j.bbagen.2013.05.003, indexed in Pubmed: 23665584.

19. Braune EB, Lendahl U. Notch - a goldilocks signaling pathway in disease and cancer therapy. Discov Med. 2016; 21(115): 189-196, indexed in Pubmed: 27115169.
20. de Matos PS, Ferreira AP, de Oliveira Facuri F, et al. Usefulness of HBME-1, cytokeratin 19 and galectin-3 immunostaining in the diagnosis of thyroid malignancy. Histopathology. 2005; 47(4): 391-401, doi: 10.111 1/j.1365-2559.2005.02221.x, indexed in Pubmed: 16178894.

21. Xiang RH, Hensel CH, Garcia DK, et al. Isolation of the human semaphorin III/F gene (SEMA3F) at chromosome 3p21, a region deleted in lung cancer. Genomics. 1996; 32(1): 39-48, doi: 10.1006/geno.1996.0074, indexed in Pubmed: 8786119.

22. Xiang R, Davalos AR, Hensel CH, et al. Semaphorin $3 \mathrm{~F}$ gene from human 3p21.3 suppresses tumor formation in nude mice. Cancer Res. 2002; 62(9): 2637-2643, indexed in Pubmed: 11980661.

23. Tse C, Xiang RH, Bracht T, et al. Human Semaphorin 3B (SEMA3B) located at chromosome 3 p21.3 suppresses tumor formation in an adenocarcinoma cell line. Cancer Res. 2002; 62(2): 542-6, indexed in Pubmed: 11809707.

24. Meda C, Molla F, De Pizzol M, et al. Semaphorin 4A exerts a proangiogenic effect by enhancing vascular endothelial growth factor-A expression in macrophages. J Immunol. 2012; 188(8): 4081-4092, doi: 10.4049/jimmunol.1101435, indexed in Pubmed: 22442441.

25. Basile JR, Barac A, Zhu T, et al. Class IV semaphorins promote angiogenesis by stimulating Rho-initiated pathways through plexin-B. Cancer Res. 2004; 64(15): 5212-5224, doi: 10.1158/0008-5472.CAN-04-0126, indexed in Pubmed: 15289326.

26. Sadanandam A, Varney ML, Singh S, et al. High gene expression of semaphorin $5 \mathrm{~A}$ in pancreatic cancer is associated with tumor growth, invasion and metastasis. Int J Cancer. 2010; 127(6): 1373-1383, doi: 10.1002/ijc.25166, indexed in Pubmed: 20073063.

27. Segarra M, Ohnuki H, Maric D, et al. Semaphorin 6 A regulates angiogenesis by modulating VEGF signaling. Blood. 2012; 120(19): 4104-4115, doi: 10.1182/blood-2012-02-410076, indexed in Pubmed: 23007403.

28. Correa RG, Sasahara RM, Bengtson $\mathrm{MH}$, et al. Human semaphorin 6B [(HSA)SEMA6B], a novel human class 6 semaphorin gene: alternative splicing and all-trans-retinoic acid-dependent downregulation in glioblastoma cell lines. Genomics. 2001; 73(3): 343-348, doi: 10.1006/geno.2001.6525, indexed in Pubmed: 11350127.

29. Zhao J, Tang H, Zhao H, et al. SEMA6A is a prognostic biomarker in glioblastoma. Tumour Biol. 2015; 36(11): 8333-8340, doi: 10.1007/s13277-015-3584-y, indexed in Pubmed: 26014517.

30. Dhanabal M, Wu F, Alvarez E, et al. Recombinant semaphorin 6A-1 ectodomain inhibits in vivo growth factor and tumor cell line-induced angiogenesis. Cancer Biol Ther. 2005; 4(6): 659-668, doi: 10.4161/cbt.4.6.1733, indexed in Pubmed: 15917651.

31. Li L, Tang P, Li S, et al. Notch signaling pathway networks in cancer metastasis: a new target for cancer therapy. Med Oncol. 2017; 34(10): 180, doi: 10.1007/s12032-017-1039-6, indexed in Pubmed: 28918490.

32. Yamashita AS, Geraldo MV, Fuziwara CS, et al. Notch pathway is activated by MAPK signaling and influences papillary thyroid cancer proliferation. Transl Oncol. 2013; 6(2): 197-205, doi: 10.1593/tlo.12442, indexed in Pubmed: 23544172.

33. Piana S, Zanetti E, Bisagni A, et al. Expression of NOTCH1 in thyroid cancer is mostly restricted to papillary carcinoma. Endocr Connect. 2019; 8(8): 1089-1096, doi: 10.1530/EC-19-0303, indexed in Pubmed: 31265994.

34. Luo Z, Mu L, Zheng Y, et al. NUMB enhances Notch signaling by repressing ubiquitination of NOTCH1 intracellular domain. J Mol Cell Biol. 2020; 12(5): 345-358, doi: 10.1093/jmcb/mjz088, indexed in Pubmed: 31504682.

35. Kageyama R, Ohtsuka T, Shimojo H, et al. Dynamic Notch signaling in neural progenitor cells and a revised view of lateral inhibition. Nat Neurosci. 2008; 11(11): 1247-1251, doi: 10.1038/nn.2208, indexed in Pubmed: 18956012.

36. $\mathrm{Li} \mathrm{Li}$, Zhao F, Lu J, et al. Notch-1 signaling promotes the malignant features of human breast cancer through NF- B activation. PLoS One. 2014; 9(4): e95912, doi: 10.1371/journal.pone.0095912, indexed in Pubmed: 24760075 . 